\title{
Influence of category identity on letter matching: Conceptual penetration of visual processing or response competition?
}

\author{
Jing Chen • Robert W. Proctor
}

Published online: 10 January 2012

(C) Psychonomic Society, Inc. 2012

\begin{abstract}
Participants performed same-different matching tasks, with physical-identity instructions, on letter pairs composed from the letters $B, b$, and $p$. The letters in a pair were presented simultaneously or sequentially, with the experiments differing in whether (1) the letters could appear in two or four positions, (2) two or five SOAs were used, (3) the font was Arial or one in which the two loops of the letter $B$ were of the same size, and (4) $p$ did or did not occur in same pairs. With sequential presentation, different RTs were longer when the letters had the same name $(B b$; withincategory pair) than when they did not ( $B p$; betweencategory pair), replicating a finding by Lupyan et al. (2010). However, unlike in their study, this category effect was also significant with simultaneous presentation, tending to be nonsignificantly smaller for RTs but larger for accuracy than that obtained with sequential presentation. A similar pattern was observed when we removed a bias to respond different whenever the letter $p$ was detected in the experiments in which $p$ did not appear in same pairs. The presence of a category effect with simultaneous presentation is predicted by a response competition account, but not by Lupyan et al.'s conceptual-penetration-of-visual-processing account.
\end{abstract}

Keywords Same-different matching $\cdot$ Response competition - Continuous-flow model - Category effect . Perceptual categorization $\cdot$ Perceptual identification

From the mid-1960s through the mid-1980s, same-different matching tasks were among the chronometric methods most

J. Chen $(\bowtie) \cdot R$. W. Proctor

Department of Psychological Sciences, Purdue University,

West Lafayette, IN 47907-2081, USA

e-mail: chen548@purdue.edu widely used by cognitive psychologists to study human information processing (see, e.g., Farell, 1985; Nickerson, 1972). This is most evident in Posner's (1978) book Chronometric Explorations of Mind, in which he relies primarily on response times (RTs) obtained in matching tasks to reach conclusions about the nature of processing systems, coordination of cognitive codes, facilitation and inhibition of psychological pathways, alertness, and the capacity and control of attention. Although same-different matching is no longer the main task used to test cognitive hypotheses, it is still exploited to examine many issues (see, e.g., Kinoshita \& Kaplan, 2008; Lachmann \& van Leeuwen, 2010).

In a common version of matching tasks, participants are presented with two letters simultaneously or sequentially, to which a same or different response is to be made as quickly as possible (e.g., Krueger, 1983). Instructions can base the classification on the letters' physical identity (e.g., $A A$ would be classified as same but $A a$ as different) or name (category ${ }^{1}$ ) identity (e.g., both $A A$ and $A a$ would be same). In such tasks, same judgments are often faster than different judgments (the same-different disparity, or fast-same effect), and same judgments for two physically identical letters are faster than those for two letters that have only name identity (the name-physical disparity; see Farell, 1985; Proctor, 1981, 1986).

Recently, Lupyan, Thompson-Schill, and Swingley (2010) used a same-different matching task to examine a currently debated issue: whether conceptual categories influence visual processing (see, e.g., Lupyan, 2008). They found that, in a physical-identity matching task, participants took longer to classify pairs as different when they shared

\footnotetext{
${ }^{1}$ In the matching literature, the term "name identity" is used to refer to the "category identity" of letters, whereas Lupyan, Thompson-Schill, and Swingley (2010) used the term "conceptual category" instead. In this article, we use the three terms interchangeably to refer to the letter category.
} 
the same name $(B b)$ than when they did not $(B p)$. This effect was significant with sequential presentation but not with simultaneous presentation, leading Lupyan et al. to conclude that it was "produced by the direct influence of category knowledge on perception, rather than by a postperceptual decision bias" (p. 682). The present study evaluated the empirical and conceptual bases of their conclusion in three experiments, with the outcome being that their account, which they referred to as "conceptual penetration of visual processing," is not strongly supported. Instead, the data are more consistent with an explanation in terms of response competition (Eriksen, O’Hara, \& Eriksen, 1982), similar to the "decision-level account" against which Lupyan et al. argued.

\section{Same-different matching}

Posner and Mitchell (1967) had participants judge pairs of simultaneously presented letters as the same or different on the basis of physical- or name-identity instructions. Judgments based on physical identity were faster than those based on name identity, and under name-identity instructions, same responses were faster to physically identical pairs than to name-identical pairs (i.e., the name-physical disparity). Posner and Mitchell proposed a levels-ofprocessing account of their results, according to which a quickly formed visual code is sufficient for matching physically identical letters, but a name code that takes longer to be generated is required to match visually different letters, whether or not their names are the same. The difference in difficulty for name-identity and physical-identity judgments is evident not only in performance measures, but also in psychophysiological measures of brain activity. Palmer, Nasman, and Wilson (1994) found that the P3 component of the event-related potential was of smaller amplitude for name-identity judgments than for physical-identity judgments, consistent with the former task requiring additional processing.

In addition to levels of processing, Posner and Snyder (1975; see also Posner, 1978) attributed the name-physical disparity to pathway activation. According to this view, a stimulus will automatically activate a specific pathway in the nervous system, and processing of stimuli that share the same pathway will be facilitated. Two physically identical letters share the same pathway more than do letters that have only their name in common, resulting in a faster response. Posner and Snyder concluded, "In simultaneous matching tasks the level-of-processing component seems to dominate. In successive matching, both factors may be involved" (p. 73).

Through comparisons of results obtained with nameidentity matching tasks and letter-naming tasks, in which one of two simultaneously presented letters or the second of two sequentially presented letters had to be named, Proctor (1981) provided evidence that the primary factor accounting for the name-physical disparity in simultaneous matching tasks is indeed the level of processing at which the match can be made. In sequential matching tasks, though, the primary factor is priming, or pathway activation, which speeds encoding of the second letter when it is physically identical to the first. Proctor also provided evidence that responses on different trials are slowed by an additional factor, inhibition resulting from the two letters activating competing name codes. Further evidence implied that this competition factor was involved with both simultaneous and sequential presentations of the letters. In sum, Proctor concluded that levels of processing contribute to RT differences with simultaneous presentation of the letters, that facilitation of encoding does so with sequential presentation, and that inhibition/competition plays a role with both presentation modes.

\section{Response competition}

Eriksen, O'Hara, and Eriksen (1982) extended Proctor's (1981) model and attributed the latency differences in same-different matching to competition between the same and different responses on the basis of a continuous-flow model of visual information processing (Eriksen \& Schultz, 1979). Their account was that activation of responses occurs concurrently with the perceptual processing of stimuli, rather than taking place only after perceptual processing is completed. The percept develops gradually over time and dynamically activates various possible responses that compete with each other. The more competing responses that are activated, the longer it will take for a specific response to reach the evocation threshold.

Eriksen et al. (1982) used noise letters in addition to target letters to introduce response-compatible and incompatible conditions in a same-different matching task. They asked participants to judge the physical identity of two target letters, neglecting the noise letters, which were identical, similar, or dissimilar to the targets. Responses were faster on same trials when the noise and target were more similar, and faster on different trials when the noise and target were less similar. Unlike Proctor (1981), who attributed the different response latencies to competing naming responses, Eriksen et al. (1982) considered that the results arose from competition between the same and different responses. By this account, on the one hand, for the samedifferent disparity, the physically different letters activate not only different, but also same, responses from stimulus onset (because the two stimuli "have many features in common, particularly at the early levels of percept development"; Eriksen et al., 1982, p. 263), although the final 
decision is different. However, the physically identical letters activate only the same response, leading to a fast-same effect. On the other hand, for the name-physical disparity, along with the same response activated by the same name, the letters with only their name in common activate a different response due to their physical dissimilarity, thus delaying selection of the correct same response.

Results consistent with the response competition account have been obtained in several other studies. For example, Proctor and Healy (1985) had participants classify multiletter strings as same or different using instructions for which strings with the same letters were to be classified as same, either only if the order was the same or also if the orders were different. For pairs with the same letters but in different orders, increasing the similarity of the orders facilitated responses when those pairs were to be classified as same, but this manipulation lengthened RTs when the letters were to be classified as different. Overmyer and Simon (1985) employed nonalphabetic stimuli with two dimensions, shape and color, one defined as relevant and the other as irrelevant, in a sequential-matching task. Responses were faster when the two attributes were congruent (both could be classified as same or different) than when the two attributes were incongruent (one was same, and the other was different), which is also in agreement with the response competition view.

Studies using direct measures of response competition, such as electromyographic recordings from forearm muscles (Eriksen, Coles, Morris, \& O'Hara, 1985), dynamometer squeezes by the left or right hand (Coles, Gratton, Bashore, Eriksen, \& Donchin, 1985), and double responses with joystick movements (St. James \& Eriksen, 1991), have also supported the idea that competition occurs in the response system, not on the input side of processing. For example, St. James and Eriksen used the incidence of abortive double responses as an index of response competition and found that double responses were more frequent, not only on different than on same trials, but also on different trials for which the difference was slight than on different trials for which the difference was larger.

\section{Lupyan et al.'s (2010) study}

Lupyan et al. (2010) had participants perform a speeded physical-identity matching task to judge whether two letters - chosen from $B, b$, and $p$-were the same or different. The letter pairs were presented in two of four possible positions equidistant from a central fixation, either simultaneously (a stimulus onset asynchrony [SOA] of $0 \mathrm{~ms}$ ) or sequentially (SOA of $150,300,450$, or $600 \mathrm{~ms}$ ), with the five SOAs randomly intermixed. The pairs $B B$ and $b b$ were to be classified as same, whereas the pairs $B b$ and $B p$ were to be classified as different. Lupyan et al.'s main interest was in the latter pairs, for which the components of $B b$ were conceptually the same at the level of letter category (withincategory pair), whereas the components of $B p$ were not (between-category pair). Lupyan et al. defined the difference in RTs between the two types of pairs as the category effect. The results showed that on sequential trials, $B p$ pairs were responded to $38 \mathrm{~ms}$ faster than $B b$ pairs, but on simultaneous trials, there was only an 11-ms, nonsignificant category effect. Lupyan et al. summarized these findings as follows: "In the case of nonidentical letters, response times were longer when the stimuli were from the same conceptual category, but only when the letters were presented sequentially" (p. 682, emphasis added).

Lupyan et al. (2010) concluded that their results provided evidence for conceptual penetration of visual processing, based on the interaction between SOA and category type, which was mainly due to there being no significant category effect on simultaneous trials. The researchers emphasized this point in their General Discussion, stating, "Critically, the category effect was absent when the two pictures were presented simultaneously, and emerged over the course of a delay between the presentation of the first and second stimulus" (p. 689). Lupyan et al. proposed that, with sequential presentation, the conceptual category of the first stimulus $(B)$ feeds back into early visual processing of the second stimulus, causing it to be perceived as more similar to the first one when it is from the same category $(b)$ rather than from a different category $(p)$. In their words, "The perceived visual similarity between two stimuli will become increasingly affected by the category membership of the stimuli over the course of several hundred milliseconds, as the conceptual category information feeds back on lower-level perceptual representations" (p. 683). The greater visual similarity of the within-category stimuli is the basis for different RTs being longer to those than to the between-category stimuli.

On the other hand, Lupyan et al. (2010, p. 684) excluded what they called a "decision-level account,"

in which same-category pairs (e.g., $B b$ ) introduce a "same" response bias that competes with the desired "different" response. . . . However, in the present paradigm, neither the visual nor the conceptual response alternatives can compete until both stimuli are visible (i.e., the channel weights are effectively 0 until both stimuli are present). Thus, a decision-level account predicts a category effect that is independent of SOA.

Although we would not go so far as to say that a decision-level account, or what we are calling a "response competition account," would predict the category effect to be independent of SOA, we agree that it does predict that such an effect should be found with simultaneous as well as 
with sequential presentation. Thus, the absence of the category effect when the letters were presented simultaneously in Lupyan et al.'s (2010) study was the crucial finding that is counter to a response competition account, and thus favors an explanation in terms of the conceptual penetration of perception.

\section{Effect of category identity on performance}

In the name-identity instruction paradigm of earlier studies (see Posner, 1978, for review), the name information was relevant, as defined by the task; conversely, in the physical-identity instruction paradigm, only physical information was relevant to the classification criterion. The question, then, is how does the name (or category) information affect performance when the physical information is seemingly sufficient for the task?

The levels-of-processing view illustrated that physicalidentity matching of simultaneous stimuli can occur on a different level from name-identity matching (Posner, 1978; Posner \& Mitchell, 1967). Pachella and Miller (1976) also implied that name information is not involved in simultaneous physical-identity matching tasks, and Lupyan et al.'s (2010) proposal was that on simultaneous trials, the category knowledge of one letter does not have a chance to penetrate through visual processing of the other. In contrast, the response competition model (Eriksen et al., 1982) implies that both same and different responses are activated by the physical similarity properties from the onset of the different pairs, and that as the percept develops, details and higher-level information (including letter category) continue to activate competing responses. Thus, with simultaneous presentation, for $B b$ pairs a same response activated by name match of the two letters would interfere with a different response activated by the physical mismatch.

As mentioned, Lupyan et al. (2010) reported a nonsignificant, 11-ms category effect with simultaneous presentation. However, evidence from several other simultaneousmatching studies has suggested that this difference likely was not due to chance. With physical-identity instructions, several researchers found that RTs to different pairs tended to be about $10-20 \mathrm{~ms}$ longer if the two letters had name identity (although being physically different) than if they did not (Pachella \& Miller, 1976, Exp. 3; Posner \& Mitchell, 1967, Exp. 1; Well \& Green, 1972, Exp. 2). Finally, Besner, Coltheart, and Davelaar (1984) found, for physical-identity judgments to pairs of mixed-case letter strings, that different responses were approximately $50 \mathrm{~ms}$ longer to strings for which the only difference was that the letters were in different cases than to strings that also contained different letters in one position. Thus, on the whole, evidence does suggest that letter identity influences performance in simultaneous matching with physical-identity instructions.

\section{Present experiments}

The nonsignificant category effect on simultaneous trials in Lupyan et al.'s (2010) experiment was the key outcome that they used to argue against a response competition explanation. Yet, the data from the simultaneous-matching studies cited in the prior section show evidence consistent with a category effect. In the present study, our aim was thus to reexamine whether the effect is absent with simultaneous presentation and interacts with simultaneous/sequential presentation - as was found by Lupyan et al. - and if so, under what conditions. The overarching goal was to obtain evidence regarding whether response competition or conceptual penetration provides the best explanation of the results.

Therefore, we used a procedure similar to that of Lupyan et al.'s (2010) study, along with modified variations based on previous studies. In Experiments 1A and 1B, we used the more customary procedure of presenting letters in two constant positions, one to the left and one to the right of a central fixation, as in the prior studies that showed evidence of a category effect with simultaneous presentation. In Experiments 2A and 2B, we presented letters randomly in two of four possible positions, as in Lupyan et al.'s study. One experiment of each pair used the same range of randomly intermixed SOAs of $0,150,300,450$, and $600 \mathrm{~ms}$ that Lupyan et al. had, whereas the other used only the 0 and 450-ms SOAs, again randomly intermixed. The latter procedure allows participants to prepare maximally for the onset of the second stimulus on sequential trials. Finally, Experiment 3 was performed similarly to Experiment 2B, but with the letters $b$ and $p$ occurring equally often in same pairs, to eliminate any differential bias to respond different for pair types containing the letter $p$.

\section{Experiments 1A and 1B}

Method

\section{Participants}

A group of 48 Purdue University undergraduates, with normal or corrected-to-normal vision, participated for course credit: 24 in Experiment 1A, and 24 in Experiment $1 \mathrm{~B}$.

\section{Apparatus and stimuli}

The apparatus consisted of a personal computer and a 19-in. LCD color monitor, viewed at a distance of approximately 57 $\mathrm{cm}$. The stimuli were pairs of letters composed of the letters $B$ $\left(1.4^{\circ} \times 2.0^{\circ}\right), b\left(1.1^{\circ} \times 2.0^{\circ}\right)$, and $p\left(1.1^{\circ} \times 2.0^{\circ}\right)$ in Arial font (white on dark background), and both letters in a pair were of 
the same height and in the same vertical position, as in Lupyan et al. (2010). Also as in their study, same pairs were only $B B$ or $b b$, whereas different pairs were composed of $B b$ and $B p$. On half of the trials, the letters in a pair were presented simultaneously on each side $\left(1.2^{\circ}\right)$ of a central fixation cross $\left(0.7^{\circ} \times\right.$ $0.7^{\circ}$ ); on the other half, one letter was displayed on the left or the right side (.50 probability) for a short time before the second letter appeared on the other side. As is indicated by the statement in Lupyan et al.'s Results section that "there were no effects of presentation order (e.g., $B p$ vs. $p B$ )" (p. 684), for sequential different pairs Lupyan et al. presented the letter $B$ first on half of the trials. Consequently, we used both presentation orders for the members of the sequential different pairs, with each order occurring equally often.

In Experiment $1 \mathrm{~A}$, the SOA on sequential trials was $450 \mathrm{~ms}$, whereas in Experiment 1B, it could be 150, 300, 450 , or $600 \mathrm{~ms}$, each occurring with the same probability (i.e., each nonzero SOA occurred on $12.5 \%$ of the trials). The fixation sign was presented for $750 \mathrm{~ms}$ before the first letter or the simultaneous letter pair, and the two letters were presented until a response was made.

\section{Procedure}

Participants responded via a standard keyboard buttonpress with the left or the right index finger to indicate same, if the letters in a pair were physically identical, or different otherwise. Half of the participants were to press the LEFT key ("z") on same trials, and the RIGHT key ("/") on different trials, and the other half were assigned to the opposite response mapping. There were equal numbers of same and different trials, and within the latter trial type, equal numbers of within- and between-category trials. Participants in Experiment $1 \mathrm{~A}$ completed 32 practice trials, followed by 288 experimental trials; those in Experiment 1B completed 30 practice trials (due to two trials inadvertently being omitted), followed by 512 experimental trials, with a 5 -min break in the middle of the experiment. More trials were conducted in Experiment $1 \mathrm{~B}$ due to the increased number of SOAs. Participants were encouraged to respond as quickly and as accurately as possible. A $500-\mathrm{ms}$ blank screen was presented after each response, and an error tone $(400-\mathrm{Hz}$, $500-\mathrm{ms}$ duration) sounded during the blank interval for incorrect responses.

\section{Results}

Both RT and response accuracy measures were subjected to separate repeated measures analyses of variance (ANOVAs) as a function of SOA (1) for same versus different responses and, (2) within different responses, for within- versus between-category pairs. Separate analyses showed that the response mapping (same to a left response and different to a right response, or vice versa) did not interact with any variables of interest (all $F_{\mathrm{S}}<2$ ), nor did the order of the positions in which the letters were presented on sequential trials (first letter to the left or right; all $F_{\mathrm{S}}<1$ ).

\section{Experiment $1 \mathrm{~A}$}

Two of the participants were replaced, one because of low accuracy ( $81.4 \%$ correct responses), and the other because of overall long RTs (mean RT of 730 ms was outside the range of $\pm 2.5 S D$ s of the remaining participants' mean RTs). The mean accuracy of the other 24 participants was $97.2 \%$. Trials responded to incorrectly were excluded from analyses of the RT data. A cutoff based on each individual participant's mean and $S D$ of RTs in the simultaneous and sequential conditions was used, by which trials with RTs 2.5 $S D$ s shorter $(0.1 \%)$ or longer $(2.3 \%)$ than the mean RT were excluded. The data were also analyzed with the cutoff method used by Lupyan et al. (2010), by which trials with RTs $<200$ or $>1,200 \mathrm{~ms}$ were excluded. Similar result patterns were obtained with both cutoff methods in all the experiments of the present study, so we only report the results using the $S D$ cutoff, the means of which are shown in Tables 1 and 2 for RTs and accuracy, respectively.

Same-different analyses Overall, participants responded faster but less accurately on same trials $(M \mathrm{~s}=524 \mathrm{~ms}$ and $96.7 \%)$ than on different trials $(M \mathrm{~s}=569 \mathrm{~ms}$ and $97.7 \%), F \mathrm{~s}$ $(1,23)=55.77$ and $6.29, p \mathrm{~s} \leq .020, \eta_{\mathrm{p}}{ }^{2}=.71$ and .22 , which is a customary result pattern indicating that the fast-same effect is not due to a bias to respond same (which would yield more false same responses on different trials; Krueger, 1983; Proctor \& Rao, 1983). Responses were also faster on sequential trials than on simultaneous trials $(M \mathrm{~s}=518$ vs. $574 \mathrm{~ms}), F(1,23)=153.04, p<.001, \eta_{\mathrm{p}}{ }^{2}=.87$. There was a significant interaction between match type (same vs. different) and SOA ( 0 vs. $450 \mathrm{~ms}$ ) for both RTs and accuracy, $F \mathrm{~s}$ $(1,23)=25.12$ and $7.75, p \leq .011, \eta_{\mathrm{p}}{ }^{2}=.52$ and .25 . The RT advantage for same trials was smaller with simultaneous presentation $(M=33 \mathrm{~ms}), t(23)=4.78, p<.001$, than with sequential presentation $(M=58 \mathrm{~ms}), t(23)=9.13, p<.001$, as is often found (e.g., Posner \& Boies, 1971), whereas the accuracy advantage for different trials was larger with simultaneous $(M=1.7 \%), t(23)=2.98, p=.007$, than with sequential presentation $(M=0.2 \%), t<1$.

Within-versus between-category analyses In the different trials, responses were slower and less accurate on withincategory trials $(B b ; M \mathrm{~s}=581 \mathrm{~ms}$ and $96.6 \%)$ than on between-category trials $(B p ; M \mathrm{~s}=557 \mathrm{~ms}$ and $98.7 \%), F \mathrm{~s}(1$, 23) $=13.89$ and $11.83, p \mathrm{~s} \leq .002, \eta_{\mathrm{p}}{ }^{2}=.38$ and .34 , replicating Lupyan et al.'s (2010) finding. Also, RTs were longer when the stimuli were presented simultaneously rather than 
Table 1 RTs (in milliseconds) on different types of trials as a function of stimulus onset asynchrony in all experiments

\begin{tabular}{|c|c|c|c|c|c|c|c|}
\hline SOA & $\mathrm{BB} / \mathrm{bb}$ & $\mathrm{Bb}$ & $\mathrm{Bp}$ & SOA & $\mathrm{BB} / \mathrm{bb}$ & $\mathrm{Bb}$ & $\mathrm{Bp}$ \\
\hline & \multicolumn{4}{|c|}{ Experiment $1 \mathrm{~A}$} & \multicolumn{3}{|c|}{ Experiment $1 \mathrm{~A}$} \\
\hline 0 & 558 & 603 & 578 & 0 & 96.3 & 96.8 & 99.2 \\
\hline \multirow[t]{2}{*}{450} & 489 & 559 & 535 & 450 & 97.1 & 96.4 & 98.1 \\
\hline & \multicolumn{4}{|c|}{ Experiment 1B } & \multicolumn{3}{|c|}{ Experiment 1B } \\
\hline 0 & 553 & 581 & 566 & 0 & 96.6 & 96.3 & 98.6 \\
\hline 150 & 506 & 552 & 530 & 150 & 97.2 & 97.8 & 98.4 \\
\hline 300 & 487 & 545 & 524 & 300 & 98.1 & 98.1 & 98.3 \\
\hline 450 & 477 & 547 & 509 & 450 & 98.0 & 97.3 & 98.4 \\
\hline \multirow[t]{2}{*}{600} & 491 & 546 & 510 & 600 & 97.8 & 96.3 & 98.1 \\
\hline & \multicolumn{4}{|c|}{ Experiment $2 \mathrm{~A}$} & \multicolumn{3}{|c|}{ Experiment $2 \mathrm{~A}$} \\
\hline 0 & 608 & 649 & 627 & 0 & 95.8 & 96.4 & 97.4 \\
\hline \multirow[t]{2}{*}{450} & 571 & 616 & 586 & 450 & 96.8 & 98.3 & 98.8 \\
\hline & \multicolumn{4}{|c|}{ Experiment 2B } & \multicolumn{3}{|c|}{ Experiment 2B } \\
\hline 0 & 592 & 634 & 605 & 0 & 96.2 & 96.5 & 99.1 \\
\hline 150 & 579 & 611 & 581 & 150 & 96.8 & 95.9 & 98.2 \\
\hline 300 & 552 & 602 & 565 & 300 & 97.6 & 97.4 & 98.3 \\
\hline 450 & 552 & 599 & 551 & 450 & 97.3 & 96.7 & 98.6 \\
\hline \multirow[t]{2}{*}{600} & 547 & 600 & 553 & 600 & 97.8 & 95.8 & 98.7 \\
\hline & \multicolumn{4}{|c|}{ Experiment 3} & \multicolumn{3}{|c|}{ Experiment 3} \\
\hline 0 & 635 & 674 & 660 & 0 & 96.1 & 97.3 & 97.9 \\
\hline 150 & 612 & 662 & 647 & 150 & 96.7 & 98.4 & 98.9 \\
\hline 300 & 592 & 651 & 645 & 300 & 96.5 & 95.7 & 98.6 \\
\hline 450 & 595 & 626 & 615 & 450 & 97.9 & 96.5 & 98.9 \\
\hline 600 & 603 & 646 & 615 & 600 & 98.1 & 96.4 & 97.7 \\
\hline
\end{tabular}

sequentially $(M \mathrm{~s}=591$ vs. $547 \mathrm{~ms}), F(1,23)=75.92, p<$ $.001, \eta_{\mathrm{p}}{ }^{2}=.77$. However, the interaction between category type (within vs. between) and SOA (0 vs. $450 \mathrm{~ms}$ ) was not significant for RTs or accuracy, $F_{\mathrm{S}}<1$ (see Fig. 1 and Table 2).

\section{Experiment $1 B$}

Two of the participants were replaced, one because of low accuracy ( $82.3 \%$ correct responses) and the other because of overall long RTs (mean RT of $742 \mathrm{~ms}$ was outside the range of $\pm 2.5 S D$ s of the remaining participants' mean RTs). The mean accuracy of the other 24 participants was $97.4 \%$.

A planned orthogonal comparison of the zero SOA versus the average of the four nonzero SOAs (the same as in Lupyan et al., 2010) was used in the data analyses, to compare the simultaneous trials with the sequential ones. An additional orthogonal comparison was conducted for the four sequential SOAs. Trials responded to incorrectly were excluded from the analyses of RT data, as were trials with RTs 2.5 SDs shorter $(0 \%)$ or longer $(1.2 \%)$ than the mean RT.
Table 2 Response accuracy (as percentages) on different types of trials as a function of stimulus onset asynchrony in all experiments

Same-different analyses Overall, participants responded faster on same than on different trials $(M \mathrm{~s}=522$ vs. $553 \mathrm{~ms}), F(1,23)=83.03, p<.001, \eta_{\mathrm{p}}{ }^{2}=.78$, but in this case, with no significant difference in response accuracy $(M \mathrm{~s}=97.1 \%$ and $97.8 \%$ for same and different trials,

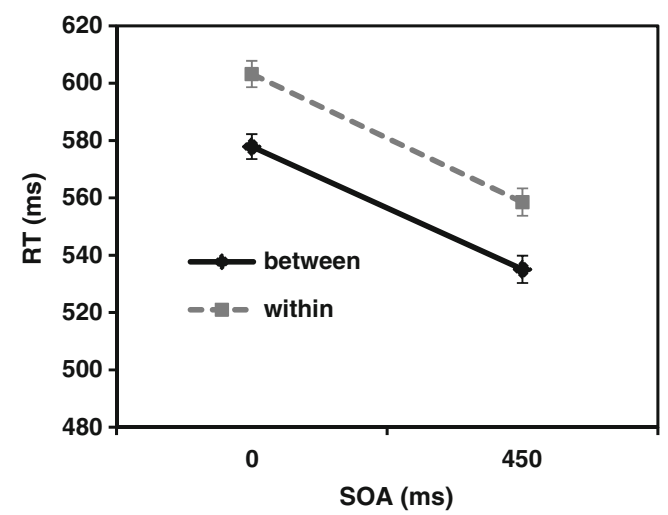

Fig. 1 Mean response times (RTs) on different trials as a function of stimulus onset asynchrony (SOA) and category type in Experiment 1A. Error bars represent \pm 1 standard error of the mean, computed using the method for within-subjects designs (Cousineau, 2005). 
respectively), $F(1,23)=1.58, p=.222, \eta_{\mathrm{p}}{ }^{2}=.06$. Responses were also faster on sequential trials than on simultaneous trials $(M \mathrm{~s}=512$ vs. $563 \mathrm{~ms}), F(1,23)=$ $157.22, p<.001, \eta_{\mathrm{p}}{ }^{2}=.87$. There was a significant interaction between match type (same vs. different) and SOA (zero vs. nonzero) for RTs, $F(1,23)=14.51, p=.001, \eta_{\mathrm{p}}{ }^{2}=.39$, but not for accuracy, $F(1,23)=1.57, p=.223, \eta_{\mathrm{p}}{ }^{2}=$. 06. As in Experiment 1A, the RT advantage for same trials was smaller with simultaneous $(M=20 \mathrm{~ms}), t(23)=4.38, p<$ .001 , than with sequential presentation $(M=42 \mathrm{~ms}), t(23)=$ $9.89, p<.001$. For the analysis comparing the four sequential SOAs, only the RT data showed significant effects: effects of match type, $F(1,23)=97.84, p<.001, \eta_{\mathrm{p}}{ }^{2}=.81$, as described in the preceding sentence, and SOA, $F(3,69)=7.89, p<.001$, $\eta_{\mathrm{p}}{ }^{2}=.26$ (with RTs decreasing as SOA increased), but no interaction, $F(3,69)=2.17, p=.099, \eta_{\mathrm{p}}^{2}=.09$.

Within-versus between-category analyses Responses were slower and less accurate on within-category trials $(M \mathrm{~s}=$ $564 \mathrm{~ms}$ and $96.8 \%)$ than on between-category trials $(M \mathrm{~s}=$ $542 \mathrm{~ms}$ and $98.5 \%), F \mathrm{~s}(1,23)=48.18$ and $22.57, p \mathrm{~s}<.001$, $\eta_{\mathrm{p}}{ }^{2}=.68$ and .52 . Also, responses were slower on simultaneous than on sequential trials $(M \mathrm{~s}=574$ vs. $533 \mathrm{~ms}), F(1$, $23)=67.11, p<.001, \eta_{\mathrm{p}}{ }^{2}=.75$. The interaction between category type (within vs. between) and SOA (zero vs. nonzero) was not significant for RTs or accuracy, $F \mathrm{~s}(1,23)=$ 2.68 and $3.61, p \mathrm{~s}=.115$ and $.070, \eta_{\mathrm{p}}{ }^{2}=.10$ and .14 (see Fig. 2 and Table 2), with the category effects being $15 \mathrm{~ms}$ and $2.3 \%$ with simultaneous presentation, $t \mathrm{~s}(23)=3.15$ and 4.73, $p \mathrm{~s} \leq .004$, as compared to $29 \mathrm{~ms}, t(23)=4.98, p<$ .001 , and $0.9 \%, t(23)=1.87, p=.075$, with sequential presentation.

For the analysis comparing the four sequential SOAs, only the RT data showed a significant effect, that of category type for RTs, $F(1,23)=24.76, p<.001, \eta_{\mathrm{p}}{ }^{2}=.52$, but no effect of SOA, $F(3,69)=2.15, p=.102, \eta_{\mathrm{p}}{ }^{2}=.09$, and no

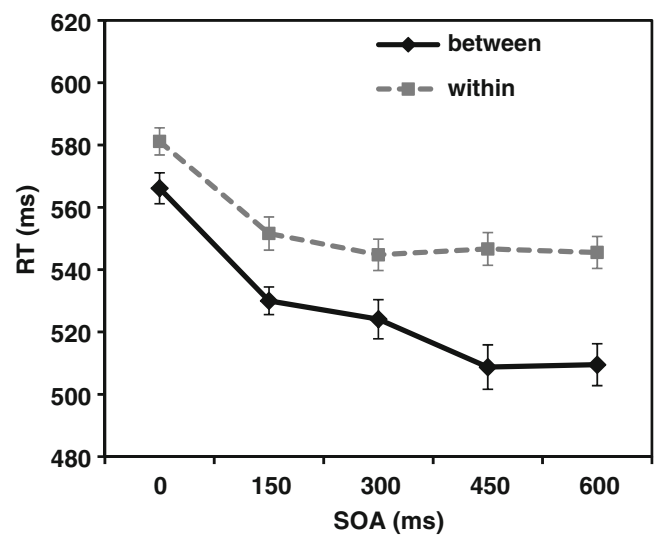

Fig. 2 Mean response times (RTs) on different trials as a function of stimulus onset asynchrony (SOA) and category type in Experiment 1B. Error bars represent \pm 1 standard error of the mean (Cousineau, 2005)
Category Type $\times$ SOA interaction, $F(3,69)=1.69, p=.177$, $\eta_{\mathrm{p}}^{2}=.07$.

\section{Discussion}

Experiments 1A and 1B replicated the commonly obtained fast-same effect (e.g., Proctor, 1981). This fast-same effect was accompanied by a preponderance of false-different errors (i.e., incorrect responses on same trials), as is often found, counter to theory that the RT advantage is due to a bias to respond same (e.g., Krueger, 1983). The fast-same effect was larger with sequential than with simultaneous presentation, as was also found in many prior studies (e.g., Posner \& Boies, 1971), although in Experiment 1A, it was countered by a larger difference in the accuracy data for simultaneous presentation than for sequential presentation.

With regard to the category effect for different pairs, similar result patterns were obtained in Experiments 1A and 1B: When the 0-SOA condition was compared with the sequential conditions, as Lupyan et al. (2010) did in their analysis, there was no significant Category Type $\times$ SOA interaction. In both subexperiments, the simultaneous trials showed significant category effects. For Experiment $1 \mathrm{~A}$, in which all sequential trials had a 450-ms SOA, the 25ms category effect with simultaneous presentation was approximately the same as the $26-\mathrm{ms}$ effect with sequential presentation. For Experiment 1B, in which SOA varied for the sequential trials, the $15-\mathrm{ms}$ category effect with simultaneous presentation tended to be smaller than the $29-\mathrm{ms}$ effect with sequential presentation, but this difference did not approach statistical significance, and category had a significant effect on response accuracy with simultaneous $(2.3 \%)$ but not with sequential $(0.9 \%)$ presentation. Thus, contrary to the conceptual-penetration account, and in agreement with the response competition account, the category effect was evident with simultaneous presentation and not significantly smaller than that with sequential presentation.

In Experiment 1B, the category effect was numerically largest at the $450-\mathrm{ms} \mathrm{SOA}(M=38 \mathrm{~ms})$ and smallest at the 0 -ms SOA $(M=15 \mathrm{~ms})$, with this difference approximating the .05 level, $t(23)=2.07$. This larger effect in RTs at the 450-ms SOA could reflect conceptual feedback on visual processing of the type proposed by Lupyan et al. (2010), if a duration of several hundred milliseconds were required for the feedback to exert an effect. However, although not statistically significant, $t(23)=1.13, p=.272$, the category effect in response accuracy tended in the opposite direction, of being larger at the $0-\mathrm{ms}$ SOA $(M=2.3 \%)$ than at the 450 ms SOA $(M=1.1 \%)$, suggesting a trade-off, about which we have more to say in the General Discussion. More 
importantly, if conceptual penetration of perception were enhancing the category effect at the 450-ms SOA in Experiment $1 \mathrm{~B}$, it also should have done so in Experiment $1 \mathrm{~A}$, in which the category effects at the 0- and 450-ms SOAs did not differ.

The substantial category effects on simultaneous trials in both versions of Experiments $1 \mathrm{~A}$ and $1 \mathrm{~B}$, which were of similar magnitudes to the effect on the sequential trials in Experiment $1 \mathrm{~A}$ and were only nonsignificantly smaller for the RT data in Experiment 1B (with the accuracy data tending to show a larger effect on simultaneous trials), differed from Lupyan et al.'s (2010) results. One difference between Experiments 1A and 1B and Lupyan et al.'s study was the stimulus display. In our experiment, the letters were presented in the same two positions, to the left and right of fixation, on every trial, whereas in their experiment the stimuli appeared in two of four positions, randomly selected for each trial. The use of two constant positions might encourage the allocation of attention to those positions, which does not occur when there is uncertainty about which of the positions will contain the letters. In Experiments $2 \mathrm{~A}$ and $2 \mathrm{~B}$, therefore, we employed a four-position display in order to determine whether the difference between simultaneous and sequential presentation would become apparent if position uncertainty were introduced, as in Lupyan et al.'s study.

\section{Experiments $2 \mathrm{~A}$ and $2 \mathrm{~B}$}

Method

\section{Participants}

The participants were 48 Purdue University students, 24 in Experiment 2A and 24 in Experiment 2B, from the same pool as in Experiment 1, but who had not taken part in that experiment.

\section{Apparatus and stimuli}

The apparatus and stimuli were the same as those in Experiment 1, except as noted. In Experiment 2A, the letters were smaller and similar in size to those of Lupyan et al. (2010) $\left[B\left(0.7^{\circ} \times 0.9^{\circ}\right) ; b\left(0.6^{\circ} \times 0.9^{\circ}\right) ; p\left(0.6^{\circ} \times 0.9^{\circ}\right)\right]$, but still in the Arial font. In Experiment 2B, the letters were in a slightly different font, that used by Lupyan et al., in which the upper and lower loops of the letter B were of the same size (see Fig. 3). ${ }^{2}$ On each trial, the letters appeared in any two of four possible locations

\footnotetext{
${ }^{2}$ We thank Gary Lupyan for providing the stimuli.
}

equidistant $\left(1.5^{\circ}\right.$ left, right, above, and below) from the central fixation cross $\left(0.4^{\circ} \times 0.4^{\circ}\right)$.

\section{Procedure}

The procedure was identical with those of Experiments 1A and $1 \mathrm{~B}$, respectively, except that participants in Experiment 2 A completed 24 practice trials followed by 384 experimental trials, and those in Experiment $2 \mathrm{~B}$ completed 48 practice trials followed by 768 experimental trials, with a 5-min break in the middle of the experiment. More trials were conducted in Experiment 2B because of the increased number of SOAs.

Results

\section{Experiment $2 \mathrm{~A}$}

Two of the participants were replaced because they had much larger standard deviations in RTs $(S D$ s $=975$ and $1,587 \mathrm{~ms}$ ) than did the remaining participants (mean $S D=$ $129 \pm 23 \mathrm{~ms}$ ). The mean accuracy of the other 24 participants was $97.1 \%$. Trials responded to incorrectly were excluded from the analyses of the RT data, as were trials with RTs $2.5 S D$ s shorter $(0.2 \%)$ or longer $(2.6 \%)$ than the participant's mean RT.

Same-different analyses Overall, participants responded faster on same trials $(M=589 \mathrm{~ms})$ than on different trials $(M=620 \mathrm{~ms}), F(1,23)=40.39, p=.001, \eta_{\mathrm{p}}{ }^{2}=.64$, but in this case with no significant difference in response accuracy $(M \mathrm{~s}=96.3 \%$ and $97.7 \%$ for same and different trials, respectively), $F(1,23)=1.46, p=.240, \eta_{\mathrm{p}}{ }^{2}=.06$. Responses were also faster on sequential than on simultaneous trials $(M \mathrm{~s}=586$ vs. $623 \mathrm{~ms}), F(1,23)=13.35$, $p=.001, \eta_{\mathrm{p}}{ }^{2}=.37$. There was no interaction between match type (same vs. different) and SOA (0 vs. $450 \mathrm{~ms}$ ) for RTs, $F<1$, or for accuracy, $F(1,23)=1.20, p=$ $.285, \eta_{\mathrm{p}}{ }^{2}=.05$.

Within- versus between-category analyses In the different trials, responses were slower on within-category trials $(M=$ $633 \mathrm{~ms})$ than on between-category trials $(M=606 \mathrm{~ms}), F(1$, $23)=35.45, p<.001, \eta_{\mathrm{p}}{ }^{2}=.61$, but in this case with no significant difference in response accuracy $(M \mathrm{~s}=97.3 \%$ and $98.1 \%$ for within- and between-category trials, respectively), $F(1,23)=3.35, p=.080, \eta_{\mathrm{p}}{ }^{2}=.13$. Responses were also slower on simultaneous than on sequential trials $(M \mathrm{~s}=638$ vs. $601 \mathrm{~ms}), F(1,23)=9.02, p=.006, \eta_{\mathrm{p}}{ }^{2}=.28$. There was no interaction between category type (within vs. between) and SOA (0 vs. $450 \mathrm{~ms}$ ) for RTs, $F(1,23)=$ 1.02, $p=.324, \eta_{\mathrm{p}}{ }^{2}=.04$, or for accuracy, $F<1$ (see Fig. 4 and Table 2). The RT advantage for between- 


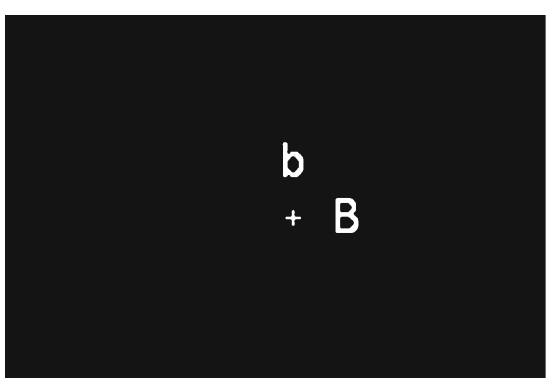

Fig. 3 Example displays of stimulus pairs from Experiments 2 and 3. The left panel shows the letters in the Arial font used in Experiment 2A (and also in Exp. 1). The right panel shows the letters used in

category trials was $22 \mathrm{~ms}$ with simultaneous presentation, $t(1,23)=4.93, p<.001$, and $30 \mathrm{~ms}$ with sequential presentation, $t(1,23)=5.84, p<.001$.

\section{Experiment $2 B$}

Three of the participants were replaced, 1 because of low accuracy (76.7\%) and the other 2 because of much larger standard deviations in RTs ( $S D \mathrm{~s}=452$ and $418 \mathrm{~ms}$ ) than the remaining participants had had (mean $S D=170 \pm 56 \mathrm{~ms}$ ). The mean accuracy of the 24 participants was $97.2 \%$. Trials responded to incorrectly were excluded from analyses of the RT data, as were trials with RTs $2.5 S D$ s shorter $(0.1 \%)$ or longer $(2.6 \%)$ than the participant's mean RT.

Same-different analyses Overall, participants responded faster and less accurately on same trials $(M \mathrm{~s}=575 \mathrm{~ms}$ and $96.8 \%)$ than on different trials $(M \mathrm{~s}=601 \mathrm{~ms}$ and $97.6 \%), F \mathrm{~s}$ $(1,23)=45.98$ and $6.46, p s \leq .018, \eta_{\mathrm{p}}{ }^{2}=.67$ and .22 . Responses were also faster on sequential than on simultaneous trials $(M \mathrm{~s}=570$ vs. $606 \mathrm{~ms}), F(1,23)=39.87, p<$ $.001, \eta_{\mathrm{p}}{ }^{2}=.63$. The interaction between match type (same vs. different) and SOA (zero vs. nonzero) was significant for

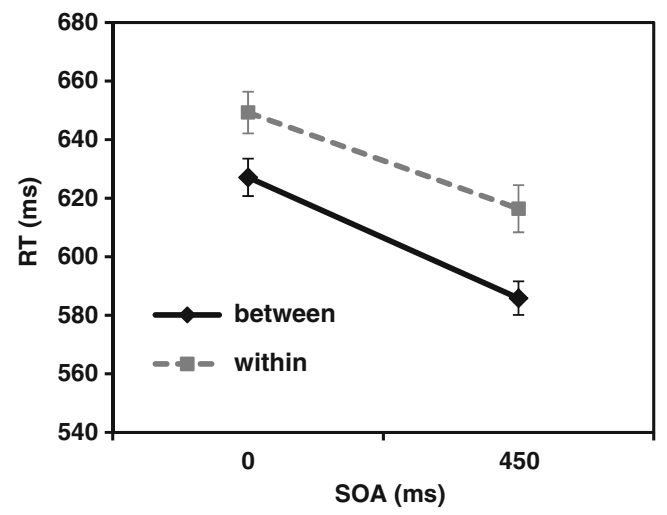

Fig. 4 Mean response times (RTs) on different trials as a function of stimulus onset asynchrony (SOA) and category type in Experiment 2A. Error bars represent \pm 1 standard error of the mean (Cousineau, 2005)

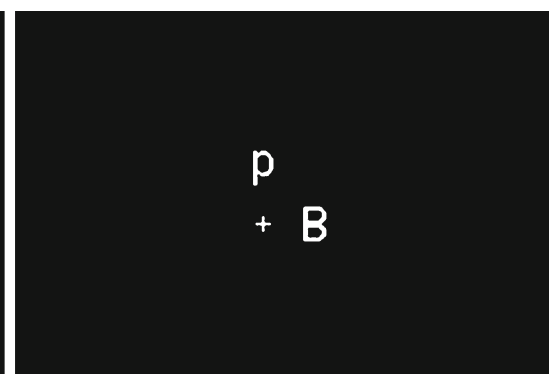

Experiments $2 \mathrm{~B}$ and 3 , in which the two loops in the letter $B$ were of the same size. For each font, the lowercase $b$ and $p$ letters were identical except for orientation

accuracy, $F(1,23)=8.44, p=.008, \eta_{\mathrm{p}}{ }^{2}=.27$, but not for RTs, $F<1$. The difference in accuracy was larger on simultaneous trials $(M=1.6 \%), t(23)=3.75, p=.001$, than on sequential trials $(M=0.1 \%), t<1$. For the analysis comparing the four sequential SOAs, only the RT data showed significant effects of match type, $F(1,23)=29.37, p<.001, \eta_{\mathrm{p}}{ }^{2}=.56$, and SOA, $F(3,69)=17.66, p<.001, \eta_{\mathrm{p}}{ }^{2}=.43$, and the interaction approached statistical significance, $F(3,69)=2.64, p=.057$, $\eta_{\mathrm{p}}^{2}=.10$.

Within-versus between-category analyses Responses were slower and less accurate on within-category trials $(M \mathrm{~s}=$ $619 \mathrm{~ms}$ and $96.5 \%)$ than on between-category trials $(M \mathrm{~s}=$ $584 \mathrm{~ms}$ and $98.8 \%), F_{\mathrm{S}}(1,23)=55.77$ and 14.92, $p \mathrm{~s} \leq .001$, $\eta_{\mathrm{p}}{ }^{2}=.72$ and .39 . They were also slower on simultaneous than on sequential trials $(M \mathrm{~s}=619$ vs. $583 \mathrm{~ms}), F(1,23)=$ $36.58, p<.001, \eta_{\mathrm{p}}{ }^{2}=.61$. The interaction between category type (within vs. between) and SOA (zero vs. nonzero) was not significant for RTs, $F(1,23)=2.84, p=.105, \eta_{\mathrm{p}}{ }^{2}=.11$, or for accuracy, $F(1,23)=4.05, p=.056, \eta_{\mathrm{p}}{ }^{2}=.15$ (see Fig. 5 and Table 2). The category effects were $29 \mathrm{~ms}$ and $2.6 \%$ with simultaneous presentation, $t \mathrm{~s}(23)=5.72$ and 3.76 , $p \mathrm{~s}<.001$, and $40 \mathrm{~ms}$ and $2.0 \%$ with sequential presentation, $t \mathrm{~s}(23)=6.70$ and $2.52, p \mathrm{~s} \leq .020$. For the

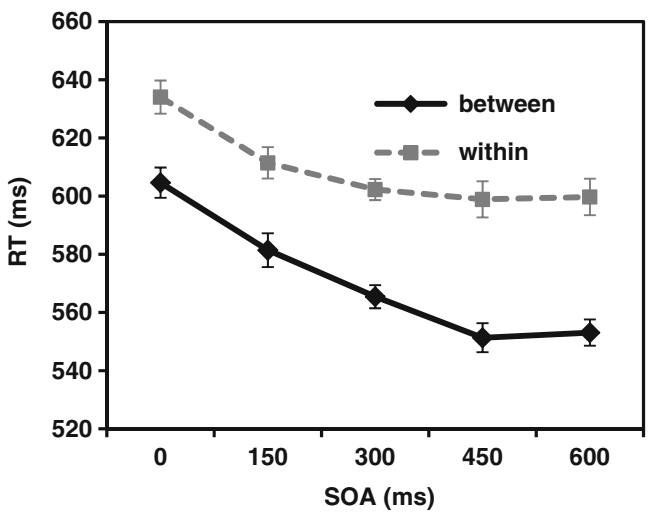

Fig. 5 Mean response times (RTs) on different trials as a function of stimulus onset asynchrony (SOA) and category type in Experiment 2B. Error bars represent \pm 1 standard error of the mean (Cousineau, 2005) 
RT analysis comparing the four sequential SOAs, we found significant main effects of category type, $F(1,23)=44.82, p<$ $.001, \eta_{\mathrm{p}}{ }^{2}=.66$, and SOA, $F(3,69)=9.27, p<.001, \eta_{\mathrm{p}}{ }^{2}=.29$, but no interaction, $F(3,69)=1.55, p=.210, \eta_{\mathrm{p}}{ }^{2}=.06$. For accuracy, only a main effect of category type was found, $F(1$, 23) $=6.35, p=.019, \eta_{\mathrm{p}}{ }^{2}=.22$.

\section{Discussion}

Experiments $2 \mathrm{~A}$ and $2 \mathrm{~B}$ showed a significant category effect with simultaneous presentation and a nonsignificant interaction with SOA. Experiment 2B closely followed the method of Lupyan et al. (2010), including the use of the same stimulus font in which the upper and lower loops of the uppercase $B$ were the same size. The font difference does not seem to be a critical factor, though, as we conducted an experiment that was the same as Experiment 2B in all respects except for using the Arial font of Experiments 1 and 2A, and the results similarly showed no reliable difference in category effect for simultaneous versus sequential presentation $(M \mathrm{~s}=30$ and $35 \mathrm{~ms}$, respectively, as compared to 29 and $40 \mathrm{~ms}$ in Exp. $2 \mathrm{~B}$, in which the accuracy data again showed a tendency for the category effect to be larger with simultaneous presentation, 2.6\%, than with sequential presentation, 2.0\%). In fact, ANOVAs of the RT and accuracy data with experiment as a variable showed no significant interactions involving experiment, with the $F$ values being less than 1.0. The Category Type $\times$ SOA interaction was not significant for RTs, $F(1,46)=$ $3.01, p=.089, \eta_{\mathrm{p}}{ }^{2}=.06$, or for accuracy, $F<1$. Thus, across Experiments 1 and 2, a significant category effect on the simultaneous trials was found with both two and four possible stimulus positions and with one or four nonzero SOAs randomly intermixed with the simultaneous trials. Moreover, in all cases, the size of the effect with simultaneous presentation was not significantly smaller than that with sequential presentation, and in the experiments with multiple nonzero SOAs, in which the category effect tended to be smaller in the RT data with simultaneous than with sequential presentation, the trend in the accuracy data was toward the effect being larger with simultaneous presentation.

A potential artifact, though, could have increased the category effect on sequential trials. Because the same trials were only $B B$ and $b b$ pairs, for trials on which the $p$ occurred as the first letter, the correct response necessarily would be different. This could have allowed participants to prepare in advance to respond different on those trials, but not on trials in which $b$ occurred first. To evaluate the extent to which the category effect on sequential trials was overestimated due to this factor, we analyzed only the different trials on which $B$ occurred first. For those trials, the sequential category effect was $15 \mathrm{~ms}$ in Experiment 2A and $17 \mathrm{~ms}$ in Experiment 2B (with no interaction across the nonzero SOAs, $F<1$ ), with both differences still being significant, $p \mathrm{~s} \leq .024$. A similar analysis showed the sequential category effects in Experiments $1 \mathrm{~A}$ and $1 \mathrm{~B}$ to be reduced to 4 and $5 \mathrm{~ms}$, ts $<1$, respectively. The fact that the effect was smaller than when the trials with the lowercase letters displayed first were included is consistent with the participants anticipating a different response when the first letter presented was $p$.

Because Lupyan et al. (2010) reported that "There were no effects of presentation order (e.g., $B p$ vs. $p B$ ), $t<1$ " (p. 684 ), we also analyzed the effect of presentation order directly for sequential within- and between-category trials. An ANOVA was conducted on the data from the first two experiments, with Presentation Order ( $p$ or $b$ occurred first vs. $B$ occurred first) and Category Type ( $B p$ vs. $B b)$ as within-subject factors, and Experiment (1A, 1B, 2A, and 2B) as a between-subjects factor. The ANOVA showed main effects of presentation order, category type, and experiment: respectively, $F_{\mathrm{S}}(1,92)=6.52,85.45$, and 5.60, $p \mathrm{~s} \leq .012$, $\eta_{\mathrm{p}}{ }^{2}=.07, .48$, and .15 . Of more interest was the significant Presentation Order $\times$ Category Type interaction, $F(1,92)=$ 28.05, $p<.001 \eta_{\mathrm{p}}{ }^{2}=.23$. RTs were $27 \mathrm{~ms}$ shorter when $p$ occurred first than when it occurred second $(M \mathrm{~s}=537$ vs. $564 \mathrm{~ms}), t(95)=4.37, p<.001$, but $6 \mathrm{~ms}$ longer when $b$ occurred first than when it occurred second ( $M \mathrm{~s}=584$ vs. $578 \mathrm{~ms}), t(95)=1.45, p=.150$. No other effects were significant, including the three-way interaction of presentation and category type with experiment, $p s>.10$. Thus, our experiments show evidence that the appearance of $p$ as the first letter biased participants to respond different faster at the onset of the second letter.

Additional evidence of such anticipation could be seen in the sequential trials, excluded as outliers, on which the RTs were 2.5 SDs shorter than the mean RT. Pooled across Experiments 1 and 2 to get a sufficient number of such trials, we found that of the 33 trials that were anticipations, 24 of those $(73 \%)$ had $p$ as the first letter. This predominance of premature responses for trials on which the first letter was $p$ was obtained despite the fact that $p$ occurred first on fewer trials than did either $b$ or $B$.

That a significant category effect of about $16 \mathrm{~ms}$ remained in Experiment 2 when only the trials on which $B$ occurred first were analyzed implies that the category effect was not entirely an artifact of the letter $p$ priming a different response when it occurred first. This value is numerically smaller than that for the simultaneous trials, but it is possible that performance on those trials was also affected by the bias to respond different if a $p$ was detected. To verify that a category effect would remain that was not artifactual, and to eliminate any influence of differential response biases on simultaneous trials caused by the letter $p$ signaling a different response, we conducted Experiment 3. In that experiment, the same pairs included both $b b$ and $p p$ (besides $B B$ ) equally often, so that no differential bias toward the different response would favor the between-category pairs. 


\section{Experiment 3}

Method

\section{Participants}

The participants were 24 Purdue University students from the same pool as in Experiments 1 and 2, but who had not taken part in the previous experiments.

\section{Apparatus, stimuli, and procedure}

The apparatus, stimuli, and procedure were the same as those aspects of Experiment 2B, including that the stimuli were exactly the same as in Lupyan et al. (2010) and the total number of trials was 768. The difference was that for the 384 same trials, half were $B B, 25 \%$ were $b b$, and $25 \%$ were $p p$. As in Experiment $2 \mathrm{~B}$, the 384 different pairs were divided equally between $B b$ and $B p$, with each letter in a pair presented first equally often on sequential trials.

\section{Results}

Two of the participants were replaced, one because of low accuracy $(88.7 \%)$ and the other because of failing to perform the experiment as instructed (singing and listening to music while doing the task). The average accuracy of the other 24 participants was $97.1 \%$. Trials with RTs 2.5 SDs shorter $(0 \%)$ or longer $(2.6 \%)$ than the participant's mean RT were excluded, as were trials responded to incorrectly.

\section{Same-different analyses}

Overall, participants responded faster on same trials $(M=$ $618 \mathrm{~ms})$ than on different trials $(M=653 \mathrm{~ms}), F(1,23)=$ $27.51, p<.001, \eta_{\mathrm{p}}{ }^{2}=.55$. Although false different responses again tended to outnumber false same responses $(M \mathrm{~s}=3.3 \%$ vs. $2.4 \%$ ), the main effect of match type was not significant for accuracy, $F(1,23)=3.83, p=.063$. Responses were also faster and more accurate on sequential than on simultaneous trials $(M \mathrm{~s}=619 \mathrm{~ms}$ and $97.5 \%$ vs. $651 \mathrm{~ms}$ and $96.8 \%), F \mathrm{~s}(1$, $23)=59.77$ and $5.13, p \mathrm{~s}<.05, \eta_{\mathrm{p}}{ }^{2}=.72$ and .18 . There was no interaction between match type (same vs. different) and SOA (zero vs. nonzero) for RTs, $F(1,23)=2.09, p=.162$, $\eta_{\mathrm{p}}{ }^{2}=.08$, but the interaction was significant for accuracy, $F$ $(1,23)=4.35, p=.048, \eta_{\mathrm{p}}{ }^{2}=.16$, with the advantage for different trials being larger with simultaneous $(M=1.5 \%), t$ $(23)=2.64, p=.015$, than with sequential $(M=0.3 \%), t<1$, presentation. For the analysis comparing the four sequential SOAs, only the RT data showed significant effects, which were main effects of match type, $F(1,23)=29.37, p<.001$, $\eta_{\mathrm{p}}{ }^{2}=.56$, and SOA, $F(3,69)=6.82, p<.001, \eta_{\mathrm{p}}{ }^{2}=.23$, as well as the interaction, $F(3,69)=3.21, p=.028, \eta_{\mathrm{p}}{ }^{2}=.12$.

Within-versus between-category analyses

Responses were 15-ms slower and 1.2\% less accurate on within-category trials $(M=660 \mathrm{~ms}$ and $97.0 \%)$ than on between-category trials $(M=645 \mathrm{~ms}$ and $98.2 \%), F_{\mathrm{s}}(1$, $23)=20.94$ and $14.92, p s \leq .001, \eta_{\mathrm{p}}{ }^{2}=.48$ and .39 . RTs were also longer on simultaneous than on sequential trials $(M \mathrm{~s}=667$ vs. $638 \mathrm{~ms}), F(1,23)=39.45, p<.001, \eta_{\mathrm{p}}{ }^{2}=.63$. The interaction between category type (within vs. between) and SOA (zero vs. nonzero) was not significant for RTs, $F<$ 1 , or for accuracy, $F(1,23)=4.05, p=.056, \eta_{\mathrm{p}}{ }^{2}=.15$ (see Fig. 6 and Table 2). With simultaneous presentation, the category effect was significant for RTs but not for accuracy $[14 \mathrm{~ms}, t(23)=2.87, p=.009 ; 0.6 \%, t(23)=1.39, p=.179]$, whereas with sequential presentation, it was significant for both RTs and accuracy [16 ms, $t(23)=3.48, p=.002 ; 1.8 \%$ less accurate for $B b$ than for $B p, t(23)=4.00, p=.001]$.

For the analysis comparing the four sequential SOAs, the RT data showed significant main effects of category type, $F$ $(1,23)=12.13, p=.002, \eta_{\mathrm{p}}{ }^{2}=.35$, and SOA, $F(3,69)=$ $6.14, p=.001, \eta_{\mathrm{p}}{ }^{2}=.21$, but no interaction, $F(1,23)=1.27$, $p=.293, \eta_{\mathrm{p}}{ }^{2}=.05$. The accuracy data showed main effects of category type, $F(1,23)=16.14, p=.001, \eta_{\mathrm{p}}{ }^{2}=.41$, and SOA, $F(3,69)=3.26, p=.027, \eta_{\mathrm{p}}{ }^{2}=.12$.

\section{Discussion}

With the method of Experiment 3, which eliminated any differential bias to respond different to the between-category pairs, a small category effect of $14 \mathrm{~ms}$ and $1.2 \%$ was evident. The 16-ms RT category effect obtained for the sequential trials converged with the 16-ms estimate for the

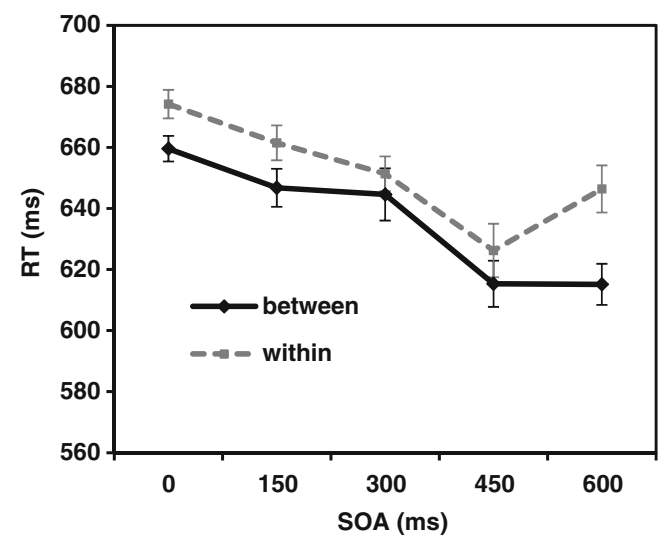

Fig. 6 Mean response times (RTs) on different trials as a function of stimulus onset asynchrony (SOA) and category type in Experiment 3. Error bars represent \pm 1 standard error of the mean (Cousineau, 2005) 
unbiased subset of trials in Experiment 2 (those on which the letter $B$ occurred first). The category effect in RTs was significant for both simultaneous and sequential trials, consistent with the response competition account and contrary to the conceptual-penetration-of-visual-processing account.

\section{General discussion}

The present study investigated how category identity influences physical-identity matching, using different letter pairs that differed only minimally (Exps. 1 and 2A) or not at all (Exps. 2B and 3) in physical similarity, but did differ in whether or not the letters were from the same letter category. As in Lupyan et al.'s (2010) study, all of our experiments revealed that between-category pairs $(B p)$ were classified as different faster than were within-category pairs $(B b)$; namely, a reliable category effect was found. However, all of the experiments, including the one most like that of Lupyan et al. (Exp. 2B), showed significant category effects with simultaneous presentation that were not significantly smaller than those with sequential presentation of the letters.

In Experiments 1 and 2, which were similar in method to Lupyan et al. (2010) in that the letter $p$ occurred only on different trials, there was a tendency for the category effect in RTs to be slightly smaller with simultaneous than with sequential presentation. We therefore conducted ANOVAs on the RT and accuracy data from Experiments 1A, 1B, 2A, and $2 \mathrm{~B}$ together, with Category Type (within vs. between) and SOA (zero vs. nonzero) as within-subject factors and Display Locations (two vs. four, or Exp. 1 vs. 2) and SOA (two vs. five, or the A subexperiments vs. the B subexperiments) as between-subjects factors. The Category Type $\times$ SOA interaction approached the .05 level for RTs, $F(1,92)=$ $3.87, p=.052, \eta_{\mathrm{p}}{ }^{2}=.04$, and accuracy, $F(1,92)=3.33, p=$ $.071, \eta_{\mathrm{p}}{ }^{2}=.04$. It did not interact with display locations or SOA condition for either measure, $F \mathrm{~s} \leq 1.31$, indicating that whether stimuli could appear in two or four locations or whether only one nonzero SOA or four were used did not matter. The pattern that the category effect for RTs tended to be smaller on simultaneous trials $(M=23 \mathrm{~ms})$ than on sequential trials $(M=31 \mathrm{~ms})$ was in the same direction as the interaction reported by Lupyan et al. (2010), although the category effect was significant with simultaneous presentation in our study but not in theirs. Note, though, that for accuracy, the category effect tended to be larger on simultaneous trials $(M=2.1 \%)$ than on sequential trials $(M=$ $1.3 \%$ ), indicating that with simultaneous presentation, some of the category effect was traded off from RTs into incorrect responses.

The data for Experiment 3, in which the letter $p$ could occur on same trials, did not display this trade-off. There was essentially no difference in the category effects for simultaneous and sequential presentation in RT, and the accuracy data tended in the opposite direction from the earlier experiments, of a slightly larger effect for sequential trials. This result suggests that the slightly smaller RT category effect with simultaneous presentation than with sequential presentation across Experiments 1 and 2 was a consequence of the letter $p$ appearing only on different trials. With simultaneous presentation, the conditional probability of a same response if a $p$ was not in the display was .67. Consequently, absence of the letter $p$ introduced a bias to respond same, which was the incorrect response for the within-category different pairs. Absence of the letter $p$ on sequential trials would have less of a biasing influence on response accuracy, because the probability of a same response when the first letter was not a $p$ was only .57 .

As was described in the Discussion section of Experiment 2, Experiments 1 and 2 showed evidence consistent with a bias to respond different for sequential trials on which $p$ occurred first, using Lupyan et al.'s (2010) method in which $p$ never appeared in a same pair. In Experiments 1 and 2, with sequential presentation, premature responses were restricted primarily to trials on which the first letter was a $p$. Also, analyzing only trials in Experiments 1 and 2 on which the letter $B$ occurred first, the category effects were reduced to 5 and $16 \mathrm{~ms}$, respectively. These results imply that there is little category effect once the response bias is removed when the letters appear in the same two positions on all trials, but there is still an effect when the letters appear in varying combinations of four positions, as in Lupyan et al.'s study.

Experiment 3 confirmed the "unbiased" estimate of the sequential category effect with a method that was similar to that of Experiment 2 but that removed the response bias by including same trials composed of instances of the letter $p$. That experiment provided evidence that the simultaneous category effect in Experiment 2 was also influenced by a bias to respond different when the letter $p$ was detected, in that its size was reduced as well in Experiment 3. Again, though, the category effect was not significantly smaller for simultaneous than for sequential trials on either the RT or accuracy measure. The category effect was evident in the mean values for both measures with both simultaneous and sequential presentation. Even though the remaining category effect was small when the response bias confound was removed, the general picture remained the same: The effect was not significantly smaller with simultaneous than with sequential presentation, contrary to what would be expected on the basis of conceptual penetration of perception.

The response competition account (Eriksen et al., 1982) treats perception as a continuous flow, which concurrently activates various responses competing with each other until one response reaches its evocation threshold. According to this model, early in the 
perceptual processing, both same and different responses are activated by the general physical information. Before the correct response is executed, more details and high-level information come into play and continue to activate the responses. Thus, according to the response competition account, relative to the pair $B p$, the letter identity of the pair $B b$ activates a same response in addition to the different response activated by the physical forms, thus leading to longer response latencies. Because this account is based on the continuous-flow model, high-level information such as letter identity should be activated gradually on simultaneous trials, predicting the category effect on those trials as well as on sequential trials.

Because the conceptual-penetration account assumes that the feedback on perception of the second letter resulting from identification of the first letter requires time to occur, the account predicts that the sequential-category effect should become larger as SOA increases. This interaction was not evident in Lupyan et al.'s (2010) data, which showed similar effect sizes for all nonzero SOAs, but it was in our Experiments $1 \mathrm{~B}$ and $2 \mathrm{~B}$, which used multiple SOAs. However, in both of those experiments, the unbiased analyses that excluded trials on which the lowercase letter occurred first showed no significant interaction of the category effect with $\mathrm{SOA}, F \mathrm{~s} \leq 1.17$. Similarly, although Experiment 3 , in which the method was unbiased, showed the largest mean category effect at the $600-\mathrm{ms} \mathrm{SOA}(31 \mathrm{~ms})$, the Category Type $\times$ SOA interaction again failed to approach significance, $F=1.27$. Thus, the SOA functions for the category effect provide little evidence for the conceptualpenetration account. Moreover, that account predicts a larger category effect for the $450-\mathrm{ms}$ SOA than for the 0-ms SOA in Experiments 1A and 2A, in which 450 ms was the only nonzero SOA used, but both experiments showed approximately the same size of category effect on simultaneous and sequential trials.

It is worth noting that a category effect of the type found in Lupyan et al.'s (2010) study and the present one does not require that the category be a learned object category or name. Lachmann and van Leeuwen $(2005,2010)$ had participants classify two sequentially presented dot patterns as the same or different on the basis of physical identity. RTs to classify pairs as different were longer when the patterns were from the same equivalence set (i.e., they were the same except for being reflected and/or different in orientation by $90^{\circ}, 180^{\circ}$, or $270^{\circ}$ ) than when the patterns were from different sets. This category effect based on visual properties likewise has been attributed to "response competition resulting from having to judge two items from the same inferred set as different" (Lachmann \& van Leeuwen, 2010, p. 295).

Although our focus has been on the category effect, the widely studied fast-same effect (Farell, 1985; Proctor, 1981) was evident in all of our experiments, with both simultaneous and sequential presentation. As we described in the introduction, evidence has implicated response competition as playing a major role in the fast-same effect (e.g., Eriksen et al., 1982). Relative to the same response, the execution of the different response is delayed because a large degree of priming is signifying same, even for different pairs. Thus, response competition not only provides a better account of the category effect than does the conceptual penetration of perception, but it relates that effect to other matching-task phenomena.

\section{Concluding remarks}

With regard to the conceptual-penetration-of-perception account, Lupyan et al. (2010) emphasized:

The critical prediction concerned the different trials. We predicted that RTs on these trials would be faster when the two stimuli were categorically different $(B p)$ than when they were categorically identical $(B b)$, but only when the first stimulus received extra processing time (i.e., when there was a nonzero stimulus onset asynchrony, or SOA).

Our results do not corroborate this critical prediction of the conceptual penetration account, that there should be a category effect only at nonzero SOAs. Rather, they show a category effect with zero SOA (i.e., simultaneous presentation) that does not differ appreciably from that found for nonzero SOAs, as predicted by the response competition account. Therefore, by Lupyan et al.'s criterion, the present results point toward response competition as the primary determinant of the category effect.

Although some aspects of our results suggest that the category effect for RTs may be slightly smaller with simultaneous than with sequential presentation, there is little reason at present to accept that feedback from the letter category on visual processing plays a significant role in the category effect. As was expressed by Norris, McQueen, and Cutler (2000), "On grounds of parsimony, if models with and without feedback can both account for a finding, the model without feedback should be chosen" (p. 324). For the conceptual-penetration account of the category effect, which relies on feedback, to be accepted over the response competition account, which does not, better evidence that uniquely implicates conceptual penetration will have to be forthcoming.

Author note We thank Yuwan Kim, Jennifer Yim, and Paul Choi for their help collecting the data, and Thomas Lachmann and Gary Lupyan for helpful comments on an earlier draft. 


\section{References}

Besner, D., Coltheart, M., \& Davelaar, E. (1984). Basic processes in reading: Computation of abstract letter identities. Canadian Journal of Psychology, 38, 126-134. doi:10.1037/h0080785

Coles, M. H., Gratton, G., Bashore, T. R., Eriksen, C. W., \& Donchin, E. (1985). A psychophysiological investigation of the continuous flow model of human information processing. Journal of Experimental Psychology: Human Perception and Performance, 11, 529-553. doi:10.1037/0096-1523.11.5.529

Cousineau, D. (2005). Confidence intervals in within-subject designs: A simpler solution to Loftus and Masson's method. Tutorials in Quantitative Methods for Psychology, 1, 42-45.

Eriksen, C. W., Coles, M. G., Morris, L. R., \& O'Hara, W. P. (1985). An electromyographic examination of response competition. Bulletin of the Psychonomic Society, 23, 165-168.

Eriksen, C. W., O’Hara, W. P., \& Eriksen, B. A. (1982). Response competition effects in same-different judgments. Perception \& Psychophysics, 32, 261-270. doi:10.3758/BF03206230

Eriksen, C. W., \& Schultz, D. W. (1979). Information processing in visual search: A continuous flow conception and experimental results. Perception \& Psychophysics, 25, 249-263. doi:10.3758/ BF03198804

Farell, B. (1985). "Same"-“different" judgments: A review of current controversies in perceptual comparisons. Psychological Bulletin, 98, 419-456. doi:10.1037/0033-2909.98.3.419

Kinoshita, S., \& Kaplan, L. (2008). Priming of abstract letter identities in the letter match task. Quarterly Journal of Experimental Psychology, 61, 1873-1885. doi:10.1080/17470210701781114

Krueger, L. E. (1983). Probing Proctor's priming principle: The effect of simultaneous and sequential presentation on same-different judgments. Journal of Experimental Psychology: Learning, Memory, and Cognition, 9, 511-523. doi:10.1037/0278-7393.9.3.511

Lachmann, T., \& van Leeuwen, C. (2005). Task-invariant aspects of goodness in perceptual representation. Quarterly Journal of Experimental Psychology, 58A, 1295-1310. doi:10.1080/ 02724980443000629

Lachmann, T., \& van Leeuwen, C. (2010). Representational economy, not processing speed, determines preferred processing strategy of visual patterns. Acta Psychologica, 134, 290-298. doi:10.1016/j. actpsy.2010.03.001

Lupyan, G. (2008). The conceptual grouping effect: Categories matter (and named categories matter more). Cognition, 108, 566-577. doi:10.1016/j.cognition.2008.03.009

Lupyan, G., Thompson-Schill, S. L., \& Swingley, D. (2010). Conceptual penetration of visual processing. Psychological Science, 21, 682-691. doi:10.1177/0956797610366099
Nickerson, R. S. (1972). Binary-classification reaction time: A review of some studies of human information-processing capabilities. Psychonomic Monograph Supplements, 17(Whole No. 65), 275 317.

Norris, D., McQueen, J. M., \& Cutler, A. (2000). Merging information in speech recognition: Feedback is never necessary. Behavioral and Brain Sciences, 23, 299-370. doi:10.1017/ S0140525X00003241

Overmyer, S. P., \& Simon, J. R. (1985). The effect of irrelevant cues on "same-different" judgments in a sequential information processing task. Acta Psychologica, 58, 237-249. doi:10.1016/00016918(85)90023-X

Pachella, R. G., \& Miller, J. O. (1976). Stimulus probability and samedifferent classification. Perception \& Psychophysics, 19, 29-34. doi:10.3758/BF03199382

Palmer, B., Nasman, V., \& Wilson, G. F. (1994). Task decision difficulty: Effects on ERPs in a same-different letter classification task. Biological Psychology, 38, 199-214. doi:10.1016/03010511(94)90039-6

Posner, M. I. (1978). Chronometric explorations of mind. Hillsdale, NJ: Erlbaum.

Posner, M. I., \& Boies, S. J. (1971). Components of attention. Psychological Review, 78, 391-408. doi:10.1037/h0031333

Posner, M. I., \& Mitchell, R. F. (1967). Chronometric analysis of classification. Psychological Review, 74, 392-409. doi:10.1037/ h0024913

Posner, M. I., \& Snyder, C. R. R. (1975). Attention and cognitive control. In R. L. Solso (Ed.), Information processing and cognition: The Loyola symposium (pp. 55-85). Hillsdale, NJ: Erlbaum.

Proctor, R. W. (1981). A unified theory for matching-task phenomena. Psychological Review, 88, 291-326. doi:10.1037/0033295X.88.4.291

Proctor, R. W. (1986). Response bias, criteria settings, and the fastsame phenomenon: A reply to Ratcliff. Psychological Review, 93, 473-477. doi:10.1037/0033-295X.93.4.473

Proctor, R. W., \& Healy, A. F. (1985). Order-relevant and orderirrelevant decision rules in multiletter matching. Journal of Experimental Psychology: Learning, Memory, and Cognition, 11, 519-537. doi:10.1037/0278-7393.11.3.519

Proctor, R. W., \& Rao, K. (1983). Evidence that the same-different disparity in letter matching is not attributable to response bias. Perception \& Psychophysics, 34, 72-76. doi:10.3758/BF03205898

St. James, J. D., \& Eriksen, C. W. (1991). Response competition produces a "fast same effect" in same-different judgments. In J. Pomerantz \& G. Lockhead (Eds.), The perception of structure (pp. 157-168). Washington, DC: American Psychological Association.

Well, A. D., \& Green, J. (1972). Effects of color differences in a letter matching task. Psychonomic Science, 29, 109-110. 